\title{
Terahertz polarization imaging
}

\author{
Nick C. J. van der Valk, Willemine A. M. van der Marel, and Paul C. M. Planken \\ Faculty of Applied Sciences, Delft University of Technology, Lorentzweg 1, 2628 CJ Delft, The Netherlands
}

Received May 31, 2005; accepted June 14, 2005

\begin{abstract}
We present a new method to measure the polarization state of a terahertz pulse by using a modified electrooptic sampling setup. To illustrate the power of this method, we show two examples in which the knowledge of the polarization of the terahertz pulse is essential for interpreting the results: spectroscopy measurements on polystyrene foam and terahertz images of a plastic coin. Both measurements show a sampleinduced rotation of the terahertz electric field vector, which is surprisingly large and is a strong function of frequency. A promising aspect of our setup is the possibility of simultaneously measuring both transversal electric field components. (C) 2005 Optical Society of America

OCIS codes: $320.0320,190.7110,260.5430$.
\end{abstract}

Terahertz time-domain spectroscopy is a powerful technique in which the time-dependent electric field of a terahertz pulse is directly measured. After the first application of the technique to imaging, ${ }^{1}$ the field rapidly expanded to include a variety of related imaging techniques, such as imaging with CCD cameras, ${ }^{2}$ terahertz tomography, ${ }^{3}$ single-shot imaging, ${ }^{4}$ and near-field imaging. ${ }^{5}$ A characteristic of these techniques is that only one component of the electric field vector is measured. This makes the images obtained with these methods sometimes difficult to interpret. A decrease in the amplitude of the measured field, for instance, is commonly interpreted as being caused by absorption or scattering. However, such a decrease could also be caused by a rotation of the electric field vector induced by a birefringence present in the sample. Besides birefringence, there are various other effects that can change the direction of a terahertz electric field, such as not-normalincidence reflection and multiple scattering. ${ }^{6}$ We note that in a recent experiment on the terahertz Hall effect a rotation of the terahertz polarization was observed when two orthogonally oriented photoconductive emitters were used. ${ }^{7}$

Here, we report a method for measuring both the direction and the length of the transversal terahertz electric field vector by using electro-optic sampling in a (111)-oriented electro-optic crystal. We demonstrate the potential of this technique in terahertz imaging and spectroscopy with two examples. In the first example, we perform spectroscopic measurements on polystyrene foam. Surprisingly, this material shows an effective birefringence, which can be measured accurately with our new technique. In the second example, we show terahertz images of a plastic coin based on a measurement of the two transversal electric field components at each pixel. The images clearly show that scattering or reflection at the edges of the coin results in a change in the polarization state of the terahertz beam.

Figure 1 shows a schematic drawing of the detection setup. We concentrate on the detection setup because the other details of our setup have been published previously. ${ }^{89}$ The terahertz electric field is measured by using the electro-optic effect, which causes a birefringence of the detection crystal propor- tional to the electric field. The birefringence causes a polarization change of the optical probe pulse, which is measured with a differential detection setup. A quarter-wave plate is placed before the ZnTe detection crystal, oriented such that the originally linear polarization of the probe beam becomes circular. A key element in our setup is the use of a $\mathrm{ZnTe}$ detection crystal with a (111) crystal orientation. This crystal orientation results in 18\% smaller signals compared with the more commonly used (110) orientation. ${ }^{10}$ However, as we discuss below, this orientation allows for the detection of both electric field components with equal sensitivities without any rotation of the detection crystal. A signal proportional to the terahertz electric field is obtained by measuring the difference in the energy in the two orthogonal polarization directions of the probe beam with a Wollaston prism and a differential detector. A half-wave plate is placed before the Wollaston prism to set the direction of the terahertz electric field to which the measurement is sensitive. Without the half-wave

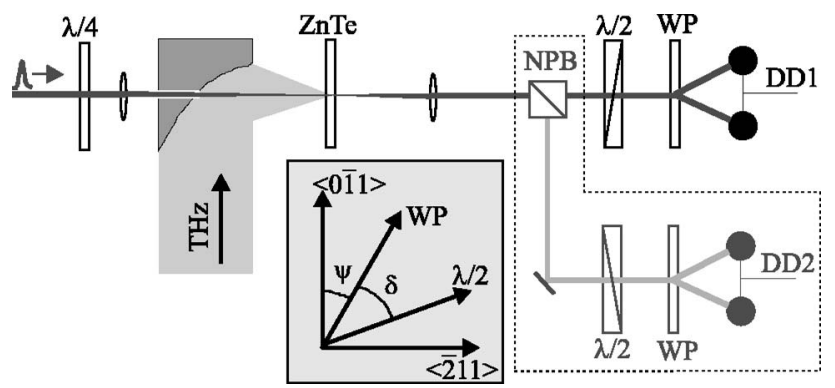

Fig. 1. Schematic of the detection setup. The terahertz beam is focused onto the ZnTe detection crystal with a parabolic mirror. The probe beam is sent through a quarter-wave plate $(\lambda / 4)$ and the parabolic mirror, and focused onto the detection crystal. After collimation, the probe beam propagates through a half-wave plate $(\lambda / 2)$ and is split into two orthogonally polarized beams by a Wollaston prism (WP). The difference in the power of the two orthogonal polarizations is measured by a differential detector (DD1). Within the dotted frame it is shown how a nonpolarizing beam splitter (NPB) can be used to create a second detection arm. The inset shows how the angles $\psi$ and $\delta$ are defined by the relative orientation of the $\langle 0 \overline{1} 1\rangle$ and $\langle\overline{2} 11\rangle$ axis of the ZnTe crystal, and the axes of the Wollaston prism and the half-wave plate. 
plate, this would require an inconvenient rotation of both the Wollaston prism and the differential detector.

The following expression is obtained for the power difference measured by the differential detector ${ }^{10}$ :

$$
\begin{aligned}
\Delta P= & \frac{\sqrt{24} \pi n^{3} r_{41} L P_{\text {tot }}}{3 \lambda}\left[E_{\overline{2} 11}^{-} \sin (2 \psi-4 \delta)\right. \\
& \left.+E_{0 \overline{1} 1} \cos (2 \psi-4 \delta)\right]
\end{aligned}
$$

where $\lambda$ is the wavelength in vacuum of the probe beam, $n$ is the refractive index of the ZnTe crystal for the probe beam, and $L$ is the thickness of the crystal. The optical power incident on the differential detector is $P_{\text {tot }}$, and $r_{41}$ is the only nonzero component of the electro-optic tensor of ZnTe. The angles $\psi$ and $\delta$ are defined in the inset in Fig. $1 . E_{2}^{-} 11$ and $E_{0 \overline{1} 1}$ are the terahertz electric fields along, respectively, the $\langle\overline{2} 11\rangle$ and $\langle 0 \overline{1} 1\rangle$ directions in the detection crystal. From Eq. (1) we can see that, either by a rotation of the half-wave plate or by a joined rotation of the Wollaston prism and the differential detector (changing $\psi$ or $\delta$ ), it is possible to decide which component of the terahertz electric field is measured by the detector.

In a measurement of two orthogonal electric field components, it is important that, when measuring one field component, the influence of the other field component be suppressed. The quality of this suppression in our setup is evaluated by measuring two orthogonal electric field components for the case in which the terahertz field is assumed to be polarized linearly. For this purpose, a wire-grid polarizer proved necessary, because our terahertz source, a photoconductive emitter, produces a slightly elliptically polarized field, as was previously reported. ${ }^{11}$ Figure $2 \mathrm{~A}$ shows the measured electric field parallel and perpendicular to the transmission direction of the polarizer. The ratio between the peak-peak values of the electric fields is 0.014 , and the ratio between the total power in the two pulses is 2552:1. Note that these numbers probably underestimate the quality of the above-mentioned suppression, as the residual field in the perpendicular direction can easily be attributed to a slight misalignment of the parabolic mirrors.

Figure 2B shows the two orthogonal electric fields measured when a $2 \mathrm{~cm}$ thick piece of polystyrene foam is placed just behind the polarizer. Besides a shift in time, there is barely any change in the parallel component of the electric field when the foam is inserted. However, the amplitude of the perpendicular component, measured after a $22.5^{\circ}$ rotation of the half-wave plate, increases dramatically. To our surprise, the strength of this component proved to be a strong function of the azimuthal orientation of the polystyrene foam, with the strongest signal plotted in Fig. 2B. Furthermore, the energy ratio in the parallel and the perpendicular components proved to be a function of frequency, as can be seen in Fig. 2(c). In this figure we plot the difference between the spectral intensities of the parallel and the perpendicular component, normalized to the sum of these intensi-
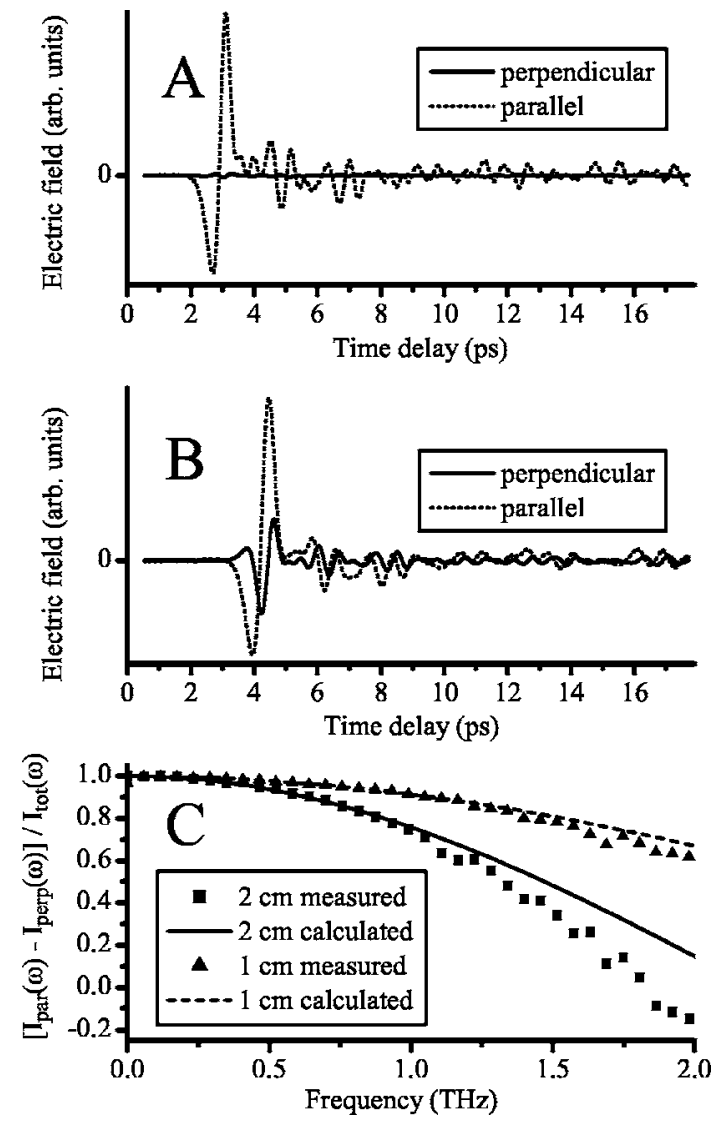

Fig. 2. A, Electric field as a function of time of the reference pulses, which traveled only through air, and, B, of the terahertz pulses after traveling through $2 \mathrm{~cm}$ polystyrene foam. The dotted and the solid lines in A and B are, respectively, the fields parallel and perpendicular to the transmission direction of the polarizer. Graph $\mathrm{C}$ shows the measured and the calculated relative intensity difference between the two polarization directions as a function of frequency for propagation through the 1 and the $2 \mathrm{~cm}$ thick pieces of polystyrene foam.

ties. The figure clearly shows that for increasing frequency an increasing part of the terahertz energy is in the perpendicular component of the electric field.

The polystyrene foam measurement can be understood in part by assuming that anisotropies induced during fabrication of the foam cause the foam to be birefringent. We can exclude surface effects as a source of anisotropy, because the observed effects increased by a factor of 2 when we doubled the thickness of the foam from 1 to $2 \mathrm{~cm}$. To verify that the signals are caused by a birefringence in the foam, we measured the time delay of the parallel component while rotating the foam around the terahertz propagation direction. The maximum changes in the time delay thus measured are $113.2 \mathrm{fs}$ for the $2 \mathrm{~cm}$ thick piece and $66.7 \mathrm{fs}$ for the $1 \mathrm{~cm}$ thick piece. When the refractive indices are assumed to be frequency independent, these time delays correspond to a refractive index difference of $1.7 \times 10^{-3}$ for the $2 \mathrm{~cm}$ piece and $2.0 \times 10^{-3}$ for the $1 \mathrm{~cm}$ piece. From these refractive index differences, we calculate the relative intensity difference between the two polarization directions as a function of frequency (solid curves, Fig. 2C). Below $1 \mathrm{THz}$ there is an excellent correspondence between 
the measurement and the calculation, which has no adjustable parameters. At higher frequencies, the agreement is somewhat worse. We note that at higher frequencies the birefringence model is no longer valid, because then the wavelength approaches the size of the bubbles in the foam, and we can no longer view the foam as an effective medium with frequency-independent refractive indices. However, the measurements are clear examples of the advantages of measuring both components of the terahertz electric field.

Our measurement technique can also be applied to the field of terahertz imaging. Figure 3 shows terahertz images obtained by measuring the transmitted terahertz electric field while scanning a plastic coin through the focus of the terahertz beam. Before this focus, a wire-grid polarizer is placed to polarize the terahertz beam. For comparison, a visible-light photograph of the coin is included [Fig. 3(c)]. The two left images show the spectral power integrated between 0.8 and $1.0 \mathrm{THz}$ for the parallel and the perpendicular component of the electric field. The images for the parallel component show distinct black lines at the edges of the coin. In contrast, the images for the perpendicular component show an enhanced power at the edges. This is consistent with a rotation of the electric field vector due to scattering and reflection at the edges of the coin. This is even clearer in Fig. 3D, which shows the angular rotation of the terahertz electric field. This rotation is obtained by calculating $\arctan \left(E_{\perp} / E_{\|}\right)$, where $E_{\perp}$ and $E_{\|}$are the spectral amplitudes between 0.8 and $1.0 \mathrm{THz}$ of, respectively, the perpendicular and the parallel electric field component. The image shows rotations of the electric field up to $45^{\circ}$. Our results clearly indicate that the features at the edges are mostly due to scattering or reflection and are not due to absorption. It would not
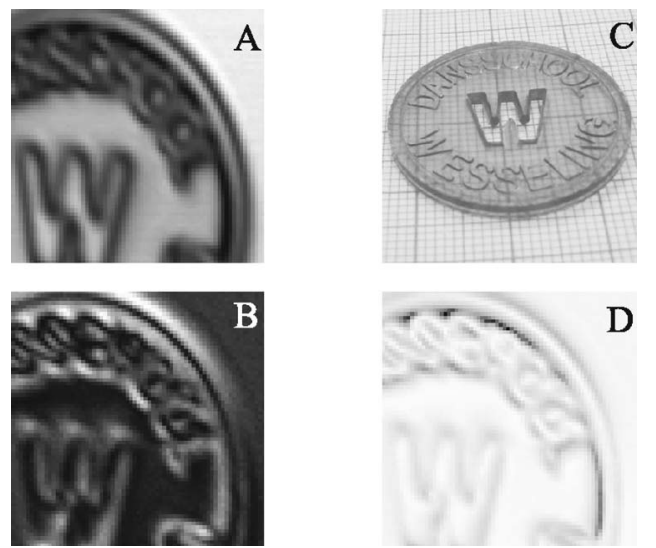

Fig. 3. A, B, D, Terahertz images and, C, visible-light photograph of a plastic coin. Left, transmitted terahertz power measured, A, parallel and, B, perpendicular to the original polarization direction (white is maximum transmission). $\mathrm{C}$, Visible-light photograph of the coin. D, Plot of the angular rotation of the direction of the terahertz electric field (white is $0^{\circ}$ rotation, black is $45^{\circ}$ rotation). have been possible to make this distinction with a measurement of only one electric field component.

Finally, we would like to point out that, although here we performed two sequential measurements of the two orthogonal polarization components, the setup can easily be extended to allow for the simultaneous measurement of the two transversal electric field components. For this purpose, an extra detection arm can be created with a nonpolarizing beam splitter and an additional half-wave plate, Wollaston prism, and differential detector, as shown in the dotted frame in Fig. 1. The two detection arms differ only in the orientation of their half-wave plates. These plates are oriented such that the two arms measure orthogonal components of the electric field. For instance, one arm measures $E_{0 \overline{1}}$, while the other simultaneously measures $E_{211}^{-}$.

In conclusion, we have presented a method to simultaneously measure the two orthogonal components of the electric field of a terahertz pulse. Key elements of this method are the use of a (111)-oriented electro-optic detection crystal and the placement of a quarter-wave plate before the crystal. By using a half-wave plate to set the direction of the measured electric-field component, we avoid cumbersome rotations of the Wollaston prism and the differential detector. We have demonstrated the applicability of our method with two examples, one related to spectroscopy and one related to imaging.

This work was performed as part of the research program of the Stichting voor Fundamenteel Onderzoek der Materie (FOM), which is financially supported by the Nederlandse Organisation voor Wetenschappelijk Onderzoek (NWO). The authors also acknowledge the support of the European Community through the IST FP6 Integrated Project TeraNova (IST-511415). N. C. J. van der Valk's e-mail address is n.c.j.vandervalk@tnw.tudelft.nl.

\section{References}

1. B. B. Hu and M. C. Nuss, Opt. Lett. 20, 807 (1995).

2. Q. Wu, T. D. Hewitt, and X.-C. Zhang, Appl. Phys. Lett. 69, 1026 (1996).

3. D. M. Mittleman, S. Hunsche, L. Boivin, and M. C. Nuss, Opt. Lett. 22, 904 (1997).

4. Z. Jiang and X.-C. Zhang, Opt. Lett. 23, 1114 (1998).

5. S. Hunsche, M. Koch, I. Brener, and M. C. Nuss, Opt. Commun. 150, 22 (1998).

6. J. Pearce, Z. Jian, and D. M. Mittleman, Opt. Lett. 29, 2926 (2004).

7. D. M. Mittleman, J. Cunningham, M. C. Nuss, and M. Geva, Appl. Phys. Lett. 71, 16 (1997).

8. G. Zhao, R. N. Schouten, N. van der Valk, W. Th. Wenckebach, and P. C. M. Planken, Rev. Sci. Instrum. 70, 1715 (2002).

9. P. C. M. Planken, H.-K. Nienhuys, H. J. Bakker, and T. Wenckebach, J. Opt. Soc. Am. B 18, 313 (2001).

10. N. C. J. van der Valk, T. Wenckebach, and P. C. M. Planken, J. Opt. Soc. Am. B 21, 622 (2004).

11. J. V. Rudd, J. L. Johnson, and D. M. Mittleman, J. Opt. Soc. Am. B 18, 1524 (2001). 